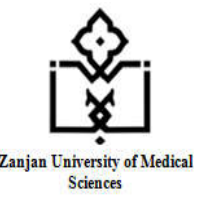

\title{
The Relationship between Cognitive-Emotional Regulation and Study Skills with Academic Procrastination in Students of the faculty of Health and Paramedicine, Zanjan University of Medical Sciences
}

\author{
Hossein Mahdion ${ }^{1}$, Qamar Kiani ${ }^{1 *}$ \\ ${ }^{1}$ Dept. of Psychology, Zanjan Branch, Islamic Azad University, Zanjan, Iran.
}

Article Info

Article Type:

Original Article

Article history:

Received 12 Nov 2016

Accepted 12 Feb 2017

Published 6 Sept 2017

Keywords:

Academic procrastination Cognitive emotion regulation Study skills

Student

\begin{abstract}
Background and Objective: Today, the prevalence of academic procrastination among students is increasing and various factors are correlated with it. Therefore, this study was conducted to determine the relationship between cognitive emotion regulation and educational skills with academic procrastination in students of the Faculty of Health and Paramedical of Zanjan University of Medical Sciences.

Materials and Methods: The research method was descriptive-correlational and the statistical population was all undergraduate students of Zanjan School of Public Health that 168 subjects (103 females, 65 males) were selected by stratified random sampling. Data were collected using standard questionnaires of Gravinsky Cognitive Censorship Management, Pulse Study and Criticism, and Solomon and Rhistlebum academic procrastination. Data were analyzed by using Pearson correlation and multiple regression tests.

Results: Normal distribution of variables was confirmed regarding to the significance level above 0.05 of Smirnov's Kolmogorov test. The results showed that there was a significant positive correlation between rumination (components of emotion regulation) and academic procrastination $(P<0.01, \mathrm{r}=0.35)$. Data showed significant negative relationship between the components of study skills, time division, physical status, reading ability, memorization, learning motivation and memory and academic procrastination $(P<0.05)$. Regression results showed that rumination, time division, physical condition, learning motivation, and memory predicted $22.1 \%, 18.8 \%, 18.1 \%, 20.9 \%$, and $20.1 \%$ of academic procrastination, respectively.

Conclusion: Designing interventions to reduce rumination, improve time division, physical status, reading ability, recording, learning motivation and memory will be effective in reducing academic procrastination.
\end{abstract}

"Corresponding Author: Qamar Kiani, Email: kherad739@yahoo.com

This article is referenced as follows:

kiani Q. The Relationship between Cognitive-Emotional Regulation and Study Skills with Academic Procrastination in Students of the faculty of Health and Paramedicine, Zanjan University of Medical Sciences. J Med Educ Dev. $2017 ; 10(26): 72-82$ 


\section{Introduction}

Procrastination is defined as postponing, delaying, or putting off a task or a decision, which is very common among high school and university students. Procrastination includes a wide range of psychological areas, and sometimes occurs unconsciously in individuals (1). Milgram introduced procrastination as an attribute or a behavioral defect manifesting itself as deferment of a task or decision (2). In addition, procrastination is known as a behavioral tendency toward postponing what is required for achieving a goal. In other words, this notion is defined as delaying a task, which has been decided to be carried out by an individual (3). According to the literature, there is a high prevalence of academic procrastination among various walks of life, especially the adolescents and youth. In a research conducted on adults, it was determined that procrastination was a medium-severe problem in life of $20 \%$ of interviewed individuals. In this regard, there is a higher rate of procrastination observed in high school and university students, who are often willing to delay their assignments, or even, obtaining their academic degree (4).

Ozer et al. marked that procrastination was observed in $40-95 \%$ of academic settings. In another research, it was reported that $70 \%$ of students and $20 \%$ of common people had tendencies toward procrastination. Ozer and Sockes also concluded that $38 \%$ of students were extremely procrastinator (5). While procrastination occurs in all different daily tasks, academic procrastination is significantly high in students, and is regarded as a decisive factor for academic success and progress (6).

Studies have shown that procrastination is not only a time-management problem, but it is also a complicated process, which includes emotional, cognitive and behavioral components. There are various theories about procrastination, each focusing on one section of this phenomenon more than others (7). Among these factors is cognitive emotion regulation, that impact of which on academic procrastination has not yet been thoroughly evaluated. In fact, cognitive emotion regulation is based on internal and external processes of responding against containment and monitoring, and evaluation and modulation of emotional interactions, especially their short-term and peculiar characteristics for achieving goals (8). Cognitive emotion regulation includes any coping strategy used by individuals when faced with extreme excitement (9). In 
addition, this concept refers to the ability to comprehend emotions, and modulate emotional experiences and expressions (10). Studies have shown that increased positive emotional experiences lead to effective dealing with stressful conditions (11). In this regard, Garnefski et al. marked that rumination is not only associated with internal self-report, but it is also related to the quality of life and general health. Moreover, it has been demonstrated that cognitive emotion regulation strategies are related to psychological distress and predict the next adjustment of an individual (12).

Another factor, which is assumed to have an impact on academic procrastination, is study skills. Many psychologists have found out that academic failure of the majority of students is not due to poor mental ability, but it is rather due to their lack of study skills. In other words, students have little information about learning and study skills (13). According to the researchers, studying is a method for coding, saving, maintaining, restating, and using information in a logical, effective, and adequate manner $(14,15)$. Results obtained by Hassan Beygi et al. (2011) have demonstrated that high $(\geq 15)$ scores of study skills (time management and procrastination, focus and memory, study aids and noting, proper strategies for test anxiety, data process and organization, motivation and attitude, survey and selection of main ideas) were more observed in students, compared to low $(<15)$ scores (16). Appropriate study methods can help students learn and memorize better and restate meanings in a more efficient way. Researchers have stated that techniques, such as dividing contents and memorizing them in accordance with the capacity of working memory, are appropriate for learning contents or putting proper gaps between the learning processes. In other word, a mass of contents cannot be memorized at once (17).

Review of previous articles led to the realization that limited number of studies have been carried out on factors affecting academic procrastination, which include cognitive emotional regulation and study skills. This lack of research is specifically observed in Iran. Therefore, it seems necessary to perform more studies, so that in addition to clarifying the concept of academic procrastination, proper solutions can be provided to reduce this issue, helping its improvement over time. With this background in mind, this study aimed to evaluate the relationship between academic procrastination of students in Health and Paramedicine 
Schools of Zanjan University of Medical Sciences, Zanjan, Iran and the variables of cognitive emotion regulation and study skills.

\section{Materials and Methods}

This descriptive and correlational research was conducted on all BSc students of Health and Paramedicine Schools of Zanjan University of Medical Sciences, including 300 individuals according to the announcement by the education office of the schools in academic year of 2015-2016. Samples were extracted using Cochran's sample size formula. In total, 168 subjects were selected considering error level of 0.05 . Data collection tools for evaluation of academic procrastination, cognitive emotion regulation and study skills were procrastination assessment scale (PAS) by Solomon and Bloom, cognitive emotion regulation questionnaire by Garnefski and study skills Inventory (PSSHI) by Palsane and Sharma, respectively. PAS was designed by Solomon and Bloom, which contains 27 items and evaluates three components, as follows: 1) preparing for exams ( 8 items, from one to eight), 2) assignment preparation (11 items, from nine to 19), and 3) preparing final articles (thesis) (eight items, from 19 to 27). Items were responded through selecting one of the alternatives of never, rarely, sometimes, often and always by the participants. In addition, questionnaires were scored on a Four-point scale (always $=4$ to never $=0$ ). However, items of 4, 6, 11, 15, 16, 21, 23, and 25 were scored reversely. Reliability of PAS was estimated at coefficient of $84 \%$ using internal consistency (18). This scale was first used in Iran by Dehghan, reliability of which was estimated at the Cronbach's alpha of 0.79 (19). In the current research, reliability of the mentioned scale was calculated at the Cronbach's alpha of 0.75.

Cognitive emotion regulation questionnaire was designed by Garnefski et al. This is a multi-dimensional and self-reporting tool, which contains 36 items and is created in adult and children forms. This questionnaire evaluates nine cognitive dimensions of selfblame, Acceptance, Rumination, Positive Refocusing, Refocus on Planning, Positive Reappraisal, Putting into Perspective, Catastrophizing, Other-blame. In this regard, four items were allocated to each dimension. Alpha coefficient for subscales of this questionnaire was reported in the range of 0.71-0.81 by Garnefski et al. In Iran, credibility of this scale was confirmed by Samani and Jokar, and its reliability was estimated at 0.79 (20). In the current study, 
reliability of the mentioned scale was calculated at Cronbach's alpha of 0.72 .

PSSHI was designed by Palsane and Sharma and contains 45 items. Respondents can answer each question by selecting one of the alternatives of "always or mostly", "sometimes" and "rarely or never". In general, the mentioned questionnaire evaluates eight areas, including division of time, physical status, ability for reading, noting, learning motivation, memory, exams, and wellness. In this regard, five items were allocated to each area. Application of this inventory is suitable for groups of 25-50 individuals. While there is no time limitation, it is better to complete the inventory in 20-25 minutes. Reliability of PSSHI was reported to be 0.88 by Kooshan and Heydari in Iran using test-retest. However, it was estimated at 0.65 applying split-half technique (21). In the present research, reliability of the mentioned inventory was estimated at the Cronbach's alpha of 0.7 .

After obtaining the required permissions from deputy of research and security of the related schools and from authorities of Schools of Health and Paramedicine to evaluate the relationship and comparison of the three variables of the research, sampling was carried out, as explained before. At first, a list of students was provided from education office of schools based on their year of enrolment, and class schedule of the students was evaluated. After the selection of one class from each year of enrolment, the researcher participated in classes and explained about goals and methods of the research and provided answer for any possible question raised by students. Following that, subjects were randomly selected and received the questionnaires. At the end of this process, 178 questionnaires were collected, 10 of which were found to be irreparably defective or incomplete, and thus were eliminated from the research. In total, 168 questionnaires were analyzed. Data analysis was performed in SPSS version 22 using descriptive statistics (mean and standard deviation), Pearson's correlation coefficient and multiple regression.

\section{Results}

In this research, $168 \mathrm{BSc}$ students from Schools of Health and Paramedicine of Zanjan University of Medical Sciences were assessed, $103(61.7 \%)$ of whom were female and $65(38.3 \%)$ were male. The highest frequency of age was related to age group of 21-24 years (42.9\%). Results related to demographic characteristics of the subjects 
are presented in Table 1. KolmogorovSmirnov test was used as default of Pearson's correlation in order to evaluate normal distribution of the variables. In this regard, results were indicative of normal distribution $(\mathrm{P}>0.05)$ (Table 2). In addition, Pearson's correlation coefficient was applied to assess the relationship between components of cognitive emotion regulation and academic procrastination of students. As observed in Table 3, a positive and significant relationship was found between only one of the components of cognitive emotion regulation (rumination) and academic procrastination $(\mathrm{P}=0.001)$.

Table 1: Demographic information of the study in terms of frequency and frequency

\begin{tabular}{|c|c|c|c|}
\hline variable & & frequency & percent \\
\hline \multirow[t]{2}{*}{ sexuality } & girl & 103 & $61 / 7$ \\
\hline & boy & 65 & $38 / 3$ \\
\hline total & & 168 & 100 \\
\hline \multirow{5}{*}{$\begin{array}{l}\text { Average grades during } \\
\text { the university }\end{array}$} & $<12$ & 13 & $7 / 7$ \\
\hline & 12-15 & 49 & $29 / 2$ \\
\hline & $15-17$ & 58 & $34 / 5$ \\
\hline & $17-20$ & 42 & 25 \\
\hline & No response & 6 & $3 / 6$ \\
\hline total & total & 168 & 100 \\
\hline \multirow[t]{5}{*}{ age } & 18-21 & 70 & $41 / 7$ \\
\hline & $21-24$ & 72 & $42 / 9$ \\
\hline & 24-30 & 17 & $10 / 1$ \\
\hline & $>\mathbf{3 0}$ & 7 & $4 / 2$ \\
\hline & No response & 2 & $1 / 2$ \\
\hline total & total & 168 & 100 \\
\hline
\end{tabular}

Table 2: Kolmogorov Smirnov test to check the variables normal

\begin{tabular}{ccc}
\hline variable & The value of the Smirnov test & The significance level \\
\hline academic Procrastination & $0 / 017$ & $0 / 085$ \\
\hline Cognitive emotion regulation & $0 / 009$ & $0 / 071$ \\
\hline Study skills & $0 / 006$ & $0 / 054$ \\
\hline
\end{tabular}


Table3: The Relationship between the Components of Cognitive Emotion Regulation and Academic Procrastination in Students

\begin{tabular}{|c|c|c|c|}
\hline \multirow{4}{*}{$\begin{array}{l}\text { Positive Cognitive } \\
\text { Emotion Regulation }\end{array}$} & \multirow[t]{2}{*}{ Positive refocusing } & The correlation coefficient & 0.150 \\
\hline & & The significance level & 0.105 \\
\hline & \multirow[t]{2}{*}{ Positive reappraisal } & The correlation coefficient & 0.131 \\
\hline & & The significance level & 0.152 \\
\hline \multirow{10}{*}{$\begin{array}{c}\text { negative Cognitive } \\
\text { Emotion Regulation }\end{array}$} & \multirow[t]{2}{*}{ Self- blame } & The correlation coefficient & 0.132 \\
\hline & & The significance level & 0.148 \\
\hline & \multirow[t]{2}{*}{ Other blame } & The correlation coefficient & 0.142 \\
\hline & & The significance level & 0.116 \\
\hline & \multirow[t]{2}{*}{ rumination } & The correlation coefficient & 0.354 \\
\hline & & The significance level & * 0.001 \\
\hline & \multirow[t]{2}{*}{ catastrophizing } & The correlation coefficient & -0.089 \\
\hline & & The significance level & 0.338 \\
\hline & \multirow[t]{2}{*}{ acceptance } & The correlation coefficient & -0.133 \\
\hline & & The significance level & 0.143 \\
\hline
\end{tabular}

In Table 4, information about relationship between study skills and academic procrastination of students are presented. According to the obtained level of significance $(P<0.01)$, a significant and negative relationship was observed between eight dimensions of study skills and academic procrastination of students, with the exception of two dimensions of exams and wellness. In
Table 5, results of multiple regression about the components of rumination, division of time, physical status, ability to reading, noting, learning motivation, memory, exams, and wellness are shown. In this regard, rumination, division of time, physical status, learning motivation, and memory predicted academic procrastination by $37.1 \%, 18.8 \%$, $18.4 \%, 23.9 \%$, and $23.1 \%$, respectively.

Table4: The Relationship between the Components of Study Skills with Academic Proclamation in Students

\begin{tabular}{ccc}
\hline \multirow{2}{*}{ Division of time } & The correlation coefficient & -0.275 \\
\cline { 2 - 3 } & The significance level & 0.002 \\
\hline \multirow{2}{*}{ Physical status } & The correlation coefficient & -0.301 \\
\cline { 2 - 3 } & The significance level & 0.001 \\
\hline \multirow{2}{*}{ notility of reading } & The correlation coefficient & -0.361 \\
\cline { 2 - 3 } & The significance level & 0.001 \\
\cline { 2 - 3 } & The correlation coefficient & -0.275 \\
\hline \multirow{2}{*}{ Learning motivation } & The significance level & 0.004 \\
\cline { 2 - 3 } & The correlation coefficient & -0.311 \\
\cline { 2 - 3 } memory & The significance level & 0.001 \\
\hline exams & The correlation coefficient & -0.327 \\
\cline { 2 - 3 } & The significance level & 0.001 \\
\hline wellness & The correlation coefficient & -0.158 \\
\cline { 2 - 3 } & The significance level & 0.084 \\
\hline & The correlation coefficient & -0.157 \\
\hline & The significance level & 0.081 \\
\hline
\end{tabular}


Table5: Multiple regressions simultaneously for predictor and criterion components

\begin{tabular}{|c|c|c|c|c|c|c|}
\hline Predictor variable & Criterion variable & B & $\begin{array}{l}\text { Standard } \\
\text { error of B }\end{array}$ & beta & $\mathrm{T}$ & $P$ value \\
\hline rumination & \multirow{7}{*}{ academic Procrastination } & 1.198 & 0.402 & 0.221 & 2.977 & 0.004 \\
\hline Division of time & & -1.056 & 0.525 & -0.188 & -2.014 & 0.047 \\
\hline Physical status & & -0.974 & 0.465 & -0.181 & -2.093 & 0.039 \\
\hline Ability of reading & & -0.304 & 0.387 & -0.075 & -0.786 & 0.434 \\
\hline noting & & -0.375 & 0.624 & -0.056 & -0.600 & 0.549 \\
\hline $\begin{array}{l}\text { Learning } \\
\text { motivation }\end{array}$ & & -1.190 & 0.461 & -0.209 & -2.583 & 0.011 \\
\hline memory & & -1.643 & 0.703 & -0.201 & -2.338 & 0.021 \\
\hline
\end{tabular}

\section{Discussion}

In the present research, the main goal was to determine the relationship between cognitive emotion regulation and study skills and academic procrastination of students in Schools of Health and Paramedicine of Zanjan University of Medical Sciences. In terms of the association between cognitive emotion regulation and academic procrastination, a significant and positive relationship was observed between only one of the components of cognitive emotion regulation (rumination) and academic procrastination. Garnefski defined rumination as continuously dwelling on one's feelings and the consequences and feelings associated with the failure without any actions to effectively correct the environment. In addition, it has been confirmed that the coping method of rumination is associated with high levels of depression. Nevertheless, this way of thinking helps the effective coping with stressful conditions to a certain extent (12).

It should be noted that the scale of cognitive emotion regulation is specifically used to show the cognitive processes applied by individuals in experiencing negative events in life. In addition, this scale has been created to better comprehend the processes affecting the emotional development of people. Therefore, it could be concluded that the more cognitive processes observed in individuals following negative events, the greater will be their level of academic procrastination. In fact, high score in the mentioned scale was indicative of effect of negative experiences on the growth development process. Referring to this definition, the positive and direct relationship of this component with academic procrastination was confirmed since constant dwelling on negative events of life increases academic procrastination in individuals. The more this component grows, the more the 
academic procrastination expands. In this regard, our findings are in line with the results obtained by Claassen, Gharabi and Motiei. In the research by Ghorabi, a positive and direct relationship was observed between cognitive emotion regulation and procrastination. Similarly, Motiei reported significant association between emotion regulation and procrastination.

Evaluation of relationship between the components of study skills and academic procrastination revealed a significant and negative relationship between academic procrastination and components of division of time, physical status, ability to reading, noting, learning motivation, and memory. To confirm this relationship, it could be stated that some measures must be taken during the period that environmental stimuli are received by sensory receptors, transferred from sensory memory to short-term memory and sent to long-term memory by coding and repeating to facilitate this process. Study skills help students learn the concepts of learning, and are in fact leaning policies and strategies for individuals. Positive communication skills of students lead to sustainable and proper learning, whereas inaccurate study skills prevent individuals from correctly understanding the contents. In addition, the type of association between the two variables demonstrated that students with academic procrastination used higher rate of inaccurate study skills, which resulted in their academic failure. Our findings are incongruence with the results obtained by Solomon and Bloom, Motiei and Shirafkan et al.. In all of the mentioned studies, a negative relationship was found between academic procrastination and study skills and their components.

\section{Conclusion}

As mentioned, a significant relationship was observed between two independent variables and one dependent variable in the current research. This relationship was positive and direct for academic procrastination and cognitive emotion regulation, whereas it was negative and reverse for academic procrastination and study skills. It is noteworthy that there was a high-rate association between academic procrastination and study skills, and study skills had a great power in predicting academic procrastination of students. Given the fact that academic procrastination is one of the most important effective factors for academic status of students today, association of which with academic failure was previously shown in studies, the importance of identifying related 
and predictive factors for academic procrastination was recognized in the present research. Therefore, academic procrastination can be avoided by strengthening cognitive emotion regulation and using proper study habits.

Over the past years, theorists of the field of psychology and education have emphasized the importance of modulation of problematic factors through effective and predictive variables, believing that application of these factors can be beneficial in indirect modification of confounding factors for academic status of students. The present study yielded considerable results in accordance with its size and level. However, the researcher mentioned lack of accuracy in control of all aspects, including control of mediating and confounding variables, which limited the generalizability of the final results. Moreover, it was impossible to compare our findings with some similar studies due to the evaluation of factors other than those assessed in the present study. This was mainly due to the wide range of factors related to procrastination. In addition, some of the students acted conservatively in completing the questionnaires, which was controlled to some extent by providing a proper environment and adhering to the confidentiality terms regarding the personal information of students.

\section{Acknowledgements}

This article was extracted from a master's thesis on psychology in Islamic Azad University of Zanjan in 2016. Hereby, we extend our gratitude to all the authorities of the university and students in Schools of Health and Paramedicine for their cooperation with the research.

\section{Reference}

1- Fernie BA, Bharucha Z, Nikčević AV, Marino C, Spada MM. A Metacognitive model of procrastination. Journal of affective disorders. 2017; 210: 196-203.

2- Savari K. The effect of training on behavior management skills, time management and reducing procrastination educational. [Dissertation], Faculty of Psychology and Educational Sciences, University of Allameh Tabatabai (RA). Tehran. 2010. [Persian]

3- Atadokht A. A study of academic procrastination based on demographic variables and its relationship with achievement motivation and academic performance of high school students. Journal of school psychology. 2015; 4(2). 135-142. [Persian]

4- Rosenthal A, Carlbring P. Group versus Internet-based cognitive-behavioral therapy for procrastination: Study protocol for a randomized 
controlled trial. JMIR Research Protocols. 2013; 2(2): e46.

5- Özer BU, Saçkes M. Effects of academic procrastination on college students' Life satisfaction. Procedia-Social and Behavioral Sciences. 2011; 12, 512-519.

6- Rosario P, Costa M, Nunez JC, et al. Academic procrastination: associations with personal, school, and family variables. The Spanish Journal of Psychology. 2009; 12, 118-127.

7- Veisi F. The relationship between self-efficacy and social anxiety in students' academic procrastination city of Marivan. [Dissertation]. senior thesis, Faculty of Psychology, Tehran University. 2012.

8- Gross JJ, Thompson RA. Emotion regulation: Conceptual foundations. Handbook of emotion regulation.second edition. New York: Guilford Press. 2015.

9- Leahy RL. Emotional schemas and resistance to change in anxiety disorders. Cognitive and behavioral Practice. 2007; 14: 35- 46.

10- Nur H, Abd Halim H. Emotion regulation and academic performance among IIUM students: A preliminary study. Journal Psikologi Malaysia. 2015; 29 (2): 81-92

11- Lingyan Li, Xiongzhao zhu, Yanjie yang, etal. Cognitive emotion regulation: characteristics and effect on quality of life in women with breast cancer. Health Qual Life Outcomes. 2015; 13: 51. 12- Legerstee JS, Garnefski N, Verhulst FC, Utens EM. Cognitive coping in anxiety-disordered adolescents. Journal of adolescence. 2011; 34(2):319-26.

13- Pindar JA. Comparative study of the effective of client central and rational emotive group counseling Models on the study habits of low achieving students [Dissertation]. Maiduguri: University of Maiduguri. 2000.

14- Chen ML. Influence of grade level on perceptual learning style preferences and language learning strategies of Taiwanese English as origin language learners. Learning and Individual Differences. 2009; 19(2). 304-308.

15- Illahi BY, Khandai H. Academic achievements and study habits of college students of district Pulwama. Journal of Education and Practice. 2015; 6(31):1-6.

16- Hassanbeigi A, Askari J, Nakhjavani M, etal. The relationship between study skills and academic performance of university students. Social and Behavioral Sciences. 2011;30: 1416 1424.

17- Benedict $\mathrm{C}$. The surprising truth about how we learn and why it happens. New York: Random House. 2015. 65-66.

18- Craswel G, poore M. Writing for academic succes. $2^{\text {nd }}$ Edition.SAGE Publications Ltd 2012.

19- Hosseinchari M, Dehghani Y. Predict academic procrastination based on self-regulation strategies on learning. Journal of Research in educational systems. 2009; 2 (4). 63-75. [Persian] 20- Samani S, Jokar B. Evaluation of reliability and validity of the short form of depression, 
anxiety and stress (DASS). Journal of Social and

Human Sciences. 2007; 26:65-76. [Persian]

21- Kooshan M, Heydari A. Study habits in students of Sabzevar School of medical sciences.
Quarterly. Journal of Sabzevar University of Medical Sciences. 2007; 13(4): 185-189. [Persian] 